\title{
Determination of the size of introgressions from Triticum timopheevii Zhuk. in the recombinant lines of bread wheat cultivar Saratovskaya 29 and their association with protein and gluten content in grain
}

\author{
Shchukina L.V.*, Pshenichnikova T.A. \\ Institute of Cytology and Genetics, SB RAS, Novosibirsk, Russia \\ * email: quality@bionet.nsc.ru
}

Gluten and protein content in wheat grain are important economic attributes that determine grain quality and its end use. Molecular markers are now making it possible to elucidate the details of the genetic control of these quantitative traits. Alien and lowcultivated wheat species are the recognized sources of reinforcement of bread wheat germplasm. Previously, we studied the introgression line 821 of Saratovskaya 29 (S29) cultivar for a number of technological characteristics of grain. This line outperformed the parent cultivar in grain gluten content and had a high grain quality. The line 821 was obtained by E.B. Budashkina from crossing S29 and tetraploid species T. timopheevii Zhuk. The size and location of introgressions in 2A, 2B, and 5A chromosomes were determined by microsatellite markers. The inter-varietal substitution line S29 (821 2A) was developed to study the effect of introgression in chromosome $2 \mathrm{~A}$ on gluten and protein content in grain in the absence of the two other introgression. The line S29 (821 2A) demonstrated a higher gluten and protein percentage in grain compared to S29. The correctness of chromosome substitution was monitored using microsatellite markers. The aim of this work was to reduce the size of the introgression from $T$. timopheevii in $2 \mathrm{~A}$ chromosome while maintaining a high content of protein and gluten. For this, two backcrosses of the line S29 (821 2A) to S29 parental cultivar were performed. As was identified using molecular markers, the resulting recombinant lines carry the introgressions of different size on $2 \mathrm{~A}$ chromosome. Seven lines were selected from the offspring for further reproduction and field studies. The report will present the results of their study for protein and gluten content and other technological characteristics of grain. Acknowledgments: This work was carried out within the framework of the budget project No. 0259-2021-0018. 